\title{
Concordancia entre la composición corporal medida con un inbody 120 y un skulpt chisel en atletas de combate adolescentes
}

\section{Concordance between body composition measured with an inbody 120 and a skulpt chisel in adolescent fighting athletes}

\author{
Roberto González-Marenco ${ }^{1}$ (D); Martha Medina-Escobedo² (iD; Mariel Garrido-Balam² (D);

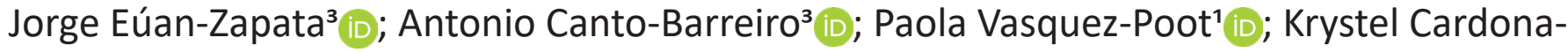 \\ Martín'(i)
}

\begin{abstract}
`Universidad Mesoamericana de San Agustín, Mérida-Yucatán-México. rob_marenco@hotmail.com; krystelcardonamartin96@gmail. com; p.m.v.p@hotmail.com

${ }^{2}$ Hospital Regional de Alta Especialidad de la Península de Yucatán-México. marthamedinaescobedo@hotmail.com

3Universidad Vizcaya de las Américas, Mérida, Yucatán, México nutriologa.mariel.garrido@hotmail.com; hagane-21@hotmail.com; antoniobarreiro-c@hotmail.com
\end{abstract}

Cómo citar: González-Marenco, R.; Medina-Escobedo, M.; Garrido-Balam, M.; Eúan-Zapata, J.; Canto-Barreiro, A.; Vasquez-Poot, P.; Cardona-Martín, K. 2021. Concordancia entre la composición corporal medida con un inbody 120 y un skulpt chisel en atletas de combate adolescentes. Rev. Digit. Act. Fis. Deport. 7(1):e1509. http://doi.org/10.31910/rdafd.v7.n1.2021.1509

Artículo de acceso abierto publicado por Revista Digital: Actividad Física y Deporte, bajo una licencia Creative Commons CC BY-NC 4.0

Publicación oficial de la Universidad de Ciencias Aplicadas y Ambientales U.D.C.A, Institución de Educación Superior Acreditada de Alta Calidad por el Ministerio de Educación Nacional.

Recibido: Julio 23 de 2020 Aceptado: Octubre 29 de 2020 Editado por: Álvaro José Gracia Díaz

\section{RESUMEN}

Introducción: La determinación de la composición corporal forma parte de la valoración morfofuncional del atleta; existiendo diferentes instrumentos para evaluarla. Objetivo: Comparar las mediciones de la composición corporal entre un InBody 120 y un Skulpt Chisel en una muestra de atletas de combate adolescentes. Metodología: Estudio cuantitativo con enfoque analítico, en el que se incluyó 24 varones $(14,2 \pm 1,9$ años, $1,6 \pm 0,1 \mathrm{~m}, 62,3 \pm 16,1 \mathrm{~kg}$, IMC $24,2 \pm 4,7 \mathrm{~kg} / \mathrm{m} 2)$. Los sujetos fueron medidos con ambos instrumentos obteniendo los valores del porcentaje de grasa corporal (\%GC), masa grasa (MG), porcentaje de masa muscular (\%MM), masa muscular (MM) y calidad muscular (CM); siguiendo las instrucciones de los fabricantes. Se utilizó la prueba de t de student para muestras relacionadas, el coeficiente de correlación de Pearson, el coeficiente de correlación concordancia de Lin y los gráficos de Bland-Altman. Se consideró significativo una $p<0,05$. Resultados: No hubo diferencia significativa entre los valores medios del \%GC $(p=0,161)$ y MG $(p=0,141)$ en la población total, pero si en la MG de los taekwondogas $(p=0,042)$. El \%GC y MG correlacionaron de manera positiva significativa entre ambos equipos de medición ( $r=0,898$ y 0,959, de manera respectiva, $p<0,01)$, similar para \%MM y CM $(r=0,771, p<0,01)$ pero no para MM y CM $(r=-0,116$, $p=0,58)$. Se encontró una concordancia pobre para, el \%GC (CCC=0,88, IC95\%=0,75-0,94) y moderada para $M G(C C C=0,95, I C 95 \%=0,89-0,97)$, además los 
gráficos de Bland-Altman mostraron variaciones individuales clínicamente relevantes para ambas variables ( $>+/-5 \%$ y $>+/-3 \mathrm{~kg}$, de manera respectiva). Conclusiones: Se concluye que las mediciones realizadas por ambos instrumentos presentaron correlaciones elevadas, pero no son concordantes ni intercambiables.

Palabras clave: Porcentaje de Grasa, Masa Grasa, Porcentaje de Mùsculo, Masa Muscular, Calidad Muscular, Impedancia Bioeléctrica.

\section{ABSTRACT}

Introduction: The determination of body composition is part of the athlete's morphofunctional assessment; there are different instruments to evaluate it. Objective: To compare the measurements of body composition between an InBody 120 and a Skulpt Chisel in a group of adolescents' combat athletes. Methodology: Quantitative study with an analytical approach, in which 24 males $(14,2 \pm 1$.9years, $1,6 \pm 0,1 \mathrm{~m}, 62,3 \pm 16,1 \mathrm{~kg}, \mathrm{BMI} 24,2 \pm 4,7 \mathrm{~kg} / \mathrm{m} 2$ ) were included. The subjects were measured with both instruments obtaining the values of the percentage of body fat (\%BF), fat mass (FM), percentage of muscle mass (\%MM), muscle mass (MM) and muscle quality (MQ); following the manufacturer's instructions. Student's t-test for paired samples, Pearson correlation coefficient, Lin's concordance correlation coefficient and Bland-Altman plots were used. Statistical significance was defined as $p<0,05$. Results: We did not observe a significant difference between $\% B F$ mean values $(p=0,161)$ and FM mean values $(p=0,141)$ in the population at large, but we did see a difference in the FM of Tae Kwon Do practitioners $(p=0,042)$. \%BF and FM showed a significant positive correlation between the two teams ( $r=0,898$ y 0,959 , respectively; $p<0,01)$, similar to \%MM and $\mathrm{MQ}(r=0,771, p<0,01)$ but not in the case of MM and QM ( $r=-0,116, p=0,58)$. We found poor concordance in the case of \%BF (CCC $=0,88$, IC95\% $=0,75-0,94)$ and moderate concordance for FM (CCC $=0,95, \quad$ IC95\%=0,89-0,97); furthermore, the Bland-Altman plots showed clinically relevant individual variations for both variables $(\geq+/-$ $5 \%$ and $\geq+/-3 \mathrm{~kg}$, respectively). Conclusions: We conclude that measurements obtained with the two instruments were highly correlated but they are neither concordant nor interchangeable.
Keywords: Fat Percentage, Fat Mass, Muscle Percentage, Muscle Mass, Muscle Quality, Bioelectric Impedance.

\section{INTRODUCCIÓN}

El estudio de la composición corporal forma parte de la evaluación morfofuncional del atleta. Este indicador nos permite conocer la respuesta fisiológica a las cargas de entrenamiento, además del estado de salud del atleta. Un programa de entrenamiento y/o una intervención nutricional pueden modificar la composición corporal, sin embargo, el peso corporal total del atleta no varía, por lo que evaluar solo la masa corporal total no es suficiente en estos sujetos (Mesa et al. 2015).

Entre los aspectos importantes del trabajo en nutrición deportiva, se encuentra la modificación del peso y la composición corporal. Muchos atletas necesitan minimizar el peso y la grasa corporal (BF) para mejorar los aspectos biomecánicos o de puntuación en los deportes de apreciación visual y estética, mientras que otros necesitan aumentar el peso y la masa muscular para mejorar el rendimiento (Peniche \& Boullosa, 2011).

Los atletas de combate se caracterizan por presentar una composición corporal con bajo contenido de grasa y un mayor desarrollo muscular, que se relaciona con una mayor fuerza, potencia, flexibilidad y una mayor capacidad anaerobia y aerobia (Franchini et al. 2007; Tabben et al. 2014). Por lo tanto, a pesar de que las categorías competitivas en los deportes de combate se dividen según el peso corporal, con el objetivo de garantizar la igualdad física y promover competiciones más justas; un atleta con una mejor composición corporal que sus homólogos tendrá más posibilidades de éxito dentro de una misma categoría (Mata-Ordoñez et al. 2018).

Evaluar adecuadamente a un atleta es muy útil e importante en estos deportes, pues le permite al profesional de nutrición saber si un atleta realmente debería perder peso (grasa corporal) o no, ya que puede haber atletas que exceden el límite superior de su categoría debido a un mayor desarrollo muscular, teniendo que cambiar la categoría de atleta (Peniche \& Boullosa, 2011). 
Los componentes principales del organismo humano están organizados en cinco niveles jerárquicos según su connotación biológica. Estos son los niveles atómico, molecular, celular, tisular y global (Wang et al. 1992; Porbén \& Borrás, 2003) Para la nutrición aplicada al deporte, la evaluación a nivel de tejido es la más interesante, ya que nos permite dividir el cuerpo en cinco componentes: tejido adiposo, tejido muscular esquelético, tejido óseo, órganos y vísceras y tejido residual (Peniche \& Boullosa, 2011).

Actualmente, el único método que permite analizar por separado todos y cada uno de los elementos que componen el organismo humano es la disección de cadáveres. Es, por eso, que se reconoce como el método de referencia a partir del cual se validan los métodos indirectos (Garrow, 1982). Sin embargo, por razones obvias, es imposible realizar este método en sujetos vivos.

Por otro lado, los métodos indirectos para la evaluación de la composición corporal no realizan una manipulación directa de los tejidos que se analizan, lo que permite un análisis in vivo. Entre los más utilizados en investigación se encuentran la tomografía computarizada, la resonancia magnética nuclear, la absorciometría dual de rayos $X$ (DXA) y la plestimografía (Costa, 2015). Estos métodos se validan teniendo como referencia el método directo o la densitometría, permitiendo medir o estimar los tejidos corporales. A pesar de su alta confiabilidad, son poco accesibles en la práctica clínica, debido principalmente, a su alto costo (Sant'anna, 2009).

La impedancia bioeléctrica (BIA) es un método doblemente indirecto, ya que ha sido validado a partir de métodos indirectos, que se utiliza con frecuencia en la práctica profesional diaria debido a su simplicidad, seguridad, facilidad de interpretación y menor costo (Sant'anna, 2009; Costa et al. 2015). La BIA es una técnica rápida y no invasiva que se basa en el principio de que la conductividad eléctrica del agua corporal varía en diferentes compartimentos, por lo que este método mide la impedancia a una pequeña corriente eléctrica aplicada a medida que pasa por el cuerpo (Lee \& Gallagher, 2008; Alvero et al. 2011). La masa libre de grasa (FFM) tiene una buena conductividad eléctrica debido a su alta concentración de agua y electrolitos, mientras que la masa grasa (FM) no tiene buena conductividad eléctrica, por lo que la impedancia es directamente proporcional a la cantidad de grasa corporal (Costa et al. 2015).

Los valores de la BIA se convierten en valores que reflejan el agua corporal total (TBW) o el líquido extracelular para posteriormente, a través de ecuaciones, conocer la masa muscular (Lustgarten \& Fielding, 2011; Thibault et al. 2012). De esta manera, la BIA estima el TBW y el FFM y, por derivación, el $B F$, utilizando la ecuación simple basada en dos componentes (FFM en $\mathrm{kg}=$ peso total en $\mathrm{kg}-\mathrm{BF}$ en kg) (Alvero et al. 2011).

La fiabilidad y la precisión de este método dependen del tipo de instrumento, los puntos de colocación de los electrodos, el nivel de hidratación, la alimentación, el ciclo menstrual, la temperatura ambiente y la ecuación de predicción utilizada (Mattsson \& Thomas, 2006; Lee \& Gallagher, 2008). Los analizadores de composición corporal InBody han mostrado fuertes correlaciones y concordancias con los métodos de referencia en poblaciones deportistas y físicamente activas (Utter \& Lambeth, 2010; Miller et al. 2016; Montgomery et al. 2017).

Por su parte, el escáner Skulpt Chisel es un instrumento que utiliza la composición miográfica y la BIA multifrecuencia para la evaluación de la composición corporal. A diferencia de los analizadores de BIA convencionales, el Skulpt no utiliza el peso, la altura o el género de la persona para calcular sus algoritmos. Además de lo anterior, existe una gran diferencia entre las inversiones para adquirir estos equipos, ya que el Skulpt puede costar de 2 a 11 veces menos que un equipo InBody (Skulpt Performance Training System, 2019).

Sin embargo, la información disponible sobre la confiabilidad de este instrumento para evaluar la composición corporal es casi inexistente (McLester et al. 2018). Además, no hay trabajos que hayan comparado el escáner Skulpt con un dispositivo de BIA de la marca InBody.

Por lo anterior, el propósito de este estudio fue comparar los datos de composición corporal del escáner Skulpt Chisel con los del equipo de BIA InBody 120 en una muestra de atletas de combate adolescentes. 


\section{MATERIALES Y MÉTODOS}

Participantes. Se realizó un estudio cuantitativo, prospectivo, transversal de concordanciacorrelación. Mediante muestreo no probabilístico por conveniencia, se estudiaron hombres (12 a 17 años y 11 meses de edad) aparentemente sanos y con 2 o más años practicando una disciplina de combate. Los sujetos que mencionaron tener alguna enfermedad crónica o aguda reciente, amputación o alguna pieza metálica para la fijación ósea y/o marcapasos fueron excluidos.

También, se excluyó a aquellos que consumieran algún medicamento, polivitamínicos, suplementos deportivos y/o sustancias naturistas o herbolarias. Los adolescentes y sus padres firmaron una carta de consentimiento informado antes de la intervención, siguiendo los informes éticos de la declaración de Helsinki (Navalta et al. 2019).

Protocolo. Los sujetos fueron citados entre las 8:00 y 10:00hrs con un ayuno previo de 8 a 10 horas. Asistieron con ropa ligera cumpliendo con las recomendaciones para la correcta aplicación del BIA (Alvero et al. 2011). Se les pidió que evitaran el consumo de bebidas alcohólicas y cafeína, además del ejercicio físico el día anterior a las mediciones.

Se les midió la talla, con un estadiómetro portátil (modelo Avanutri 315, Avanutri Equipamentos de Avaliação Ltda, Brasil) con un rango de medición de $20-210 \mathrm{~cm}$ y una precisión de $1 \mathrm{~mm}$, siguiendo la metodología establecida por la International Society Advancement Kinanthropometry (ISAK) (MarfellJones et al. 2006).

El peso y la composición corporal, se determinaron usando el analizador multifrecuencia BIA octopolar (modelo InBody 120, Arlington Heights, IL) con un rango de medición de 5 a $250 \mathrm{~kg}$ y una precisión de $0.1 \mathrm{~kg}$. Los sujetos se colocaron de pie, sin calcetines, sobre la plataforma del analizador en contacto con los electrodos para los pies, y sostuvieron el volante mientras mantenían contacto con los electrodos para las manos. Se les indicó que mantuvieran una postura correcta y que bajaran del analizador hasta que el investigador se los indicara. Se siguieron las instrucciones del fabricante para la correcta determinación de la composición corporal (Skulpt Performance Training System, 2019). El peso corporal y los valores totales y segmentarios se obtuvieron para el porcentaje de grasa corporal (\%GC) y MG, el porcentaje de masa muscular (\%MM) y la masa muscular (MM).

Posteriormente, los sujetos fueron analizados con el escáner Skulpt Chisel (Skulpt Chisel, Skulpt Inc., EE. UU.), realizando mediciones en la parte superior (antebrazo, bíceps, tríceps, hombro, pecho, espalda superior e inferior), central (abdomen) e inferior (glúteo, cuádriceps, isquiotibiales y pantorrilla) en el lado derecho e izquierdo del cuerpo. Se obtuvo el \%GC y la calidad muscular (CM) de cada área medida y los valores totales. Las mediciones se realizaron siguiendo las instrucciones del fabricante (Skulpt Performance Training System, 2019).

Análisis estadístico. Las características generales de la población se analizaron utilizando medidas de tendencia central. Los valores promedio del \%GC y MG medidos por los dos instrumentos se compararon usando la prueba $\mathrm{t}$ para muestras relacionadas. La concordancia entre los dos instrumentos para \%GC y MG se determinó utilizando el coeficiente de correlación de Lin, utilizando los puntos de corte propuestos por el autor (>0,99 casi perfecta, 0,950,99 sustancial, 0,90-0,95 moderada y $<0,90$ pobre) (Lin, 1989); y los gráficos de Bland-Altman con el programa estadístico MedCalc. La correlación entre ambos instrumentos para la $\mathrm{CM}, \% \mathrm{MM}$ y $\mathrm{MM}$ fue determinada por el coeficiente de correlación de Pearson con el programa estadístico SPSS versión 21.0. Se consideró como significativa, el valor de $\mathrm{p}<0.05$.

\section{RESULTADOS}

Se incluyó un total de 24 adolescentes varones que practicaban taekwondo $(n=18)$ y luchas $(n=6)$. La Tabla 1 muestra las características generales de la población, observando que no hubo diferencias significativas entre el \%GC $(p=0,161)$ y la MG ( $p=$ 0,144 ) determinado por los dos instrumentos. El análisis por disciplinas mostró sólo en el grupo de luchadores una diferencia significativa $(p=0,042)$ entre la MG determinada por ambos equipos. Cuando se compararon ambas disciplinas, se observó un mayor \%MM en el tren superior derecho $(p=0,033)$, izquierdo $(p=0,034)$ y abdominal $(p=0,011)$ en los luchadores, de acuerdo al InBody. También, se observó que los atletas de taekwondo presentaron un $\% G C$ mayor $(p=0,020)$ y una CM menor $(p=0,011)$ en relación a los atletas de luchas, de acuerdo al Skulpt. 


\section{Revista Digital: Actividad Física y Deporte}

Se encontró una concordancia pobre para, el IC95\%=0,89-0,97, Gráfica 2) determinada por los dos $\% G C$ (CCC=0,88, IC95\%=0,75-0,94, Gráfica 1) y instrumentos. una concordancia moderada para MG $(C C C=0,95$,

Tabla 1. Características generales de la población y comparación de las mediciones entre InBody 120 y Skulpt Chisel mediciones entre InBody 120 y Skulpt Chisel.

\begin{tabular}{|c|c|c|c|c|c|c|c|c|c|}
\hline Variables & \multicolumn{2}{|c|}{$\begin{array}{l}\text { Población total } \\
(n=24) \\
\bar{x} \pm D E\end{array}$} & \multicolumn{2}{|c|}{$\begin{array}{c}\text { Taekwondo } \\
(n=18) \\
\bar{x} \pm D E\end{array}$} & \multicolumn{2}{|c|}{$\begin{array}{c}\text { Luchas } \\
(n=6) \\
\bar{x} \pm D E\end{array}$} & \multicolumn{3}{|c|}{ Valor P } \\
\hline Edad(años) & 14 , & $\pm 1,9$ & 14, & $\pm 1,9$ & 14 , & $\pm 1,9$ & --- & --- & --- \\
\hline Peso(kg) & 62,3 & $\pm 16,1$ & 63,7 & 13,5 & 58,2 & 23,5 & --- & --- & --- \\
\hline Talla(m) & & 0,1 & 1,6 & $\pm 0,1$ & 1,5 & $\pm 0,1$ & --- & --- & --- \\
\hline IMC $\left(\mathbf{k g} / \mathrm{m}^{2}\right)$ & 24, & $\pm 4,7$ & 23,9 & 3,01 & 23, & $+6,1$ & --- & --- & --- \\
\hline & $\begin{array}{l}\text { InBody } \\
120\end{array}$ & $\begin{array}{l}\text { Skulpt } \\
\text { Chisel }\end{array}$ & $\begin{array}{c}\text { InBody } \\
120\end{array}$ & $\begin{array}{l}\text { Skulpt } \\
\text { Chisel }\end{array}$ & $\begin{array}{c}\text { InBody } \\
120\end{array}$ & $\begin{array}{l}\text { Skulpt } \\
\text { Chisel }\end{array}$ & $\mathrm{p}^{\mathrm{a}}$ & $p^{b}$ & $p^{c}$ \\
\hline$\%$ GC & $23,9 \pm 6,9$ & $24,9 \pm 6,1$ & $25,2 \pm 6,5$ & $26,5 \pm 5,6$ & $20,3 \pm 7,5$ & $19,9 \pm 5,2$ & 0,161 & 0,087 & 0,788 \\
\hline MG & $15,4 \pm 6,9$ & $15,9 \pm 6,7$ & $16,3 \pm 5,9$ & $17,3 \pm 6,0$ & $12,7 \pm 9,3$ & $12,2 \pm 7,6$ & 0,144 & $0,042^{*}$ & 0,513 \\
\hline$\% M M$ & $42,1 \pm 4,1$ & --- & $41,4 \pm 3,9$ & --- & $44,3 \pm 4,3$ & --- & --- & -- & --- \\
\hline MM & $26,2 \pm 6,7$ & --- & $26,4 \pm 6,0$ & --- & $25,5 \pm 9,2$ & --- & --- & --- & --- \\
\hline $\mathrm{CM}$ & - & $54,5 \pm 17,2$ & & $49,5 \pm 13,6$ & -- & $69,4 \pm 19,3$ & -- & --- & --- \\
\hline
\end{tabular}

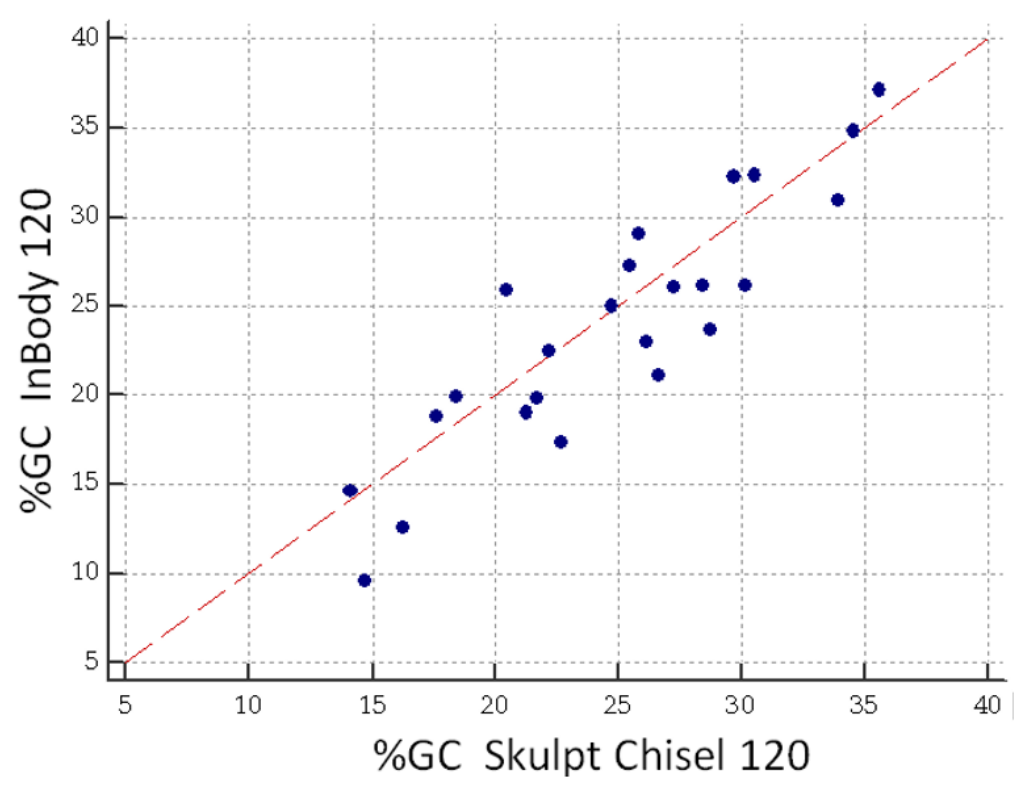

Gráfica 1. Concordancia entre InBody 120 y Skulpt Chisel para las mediciones de \%GC.

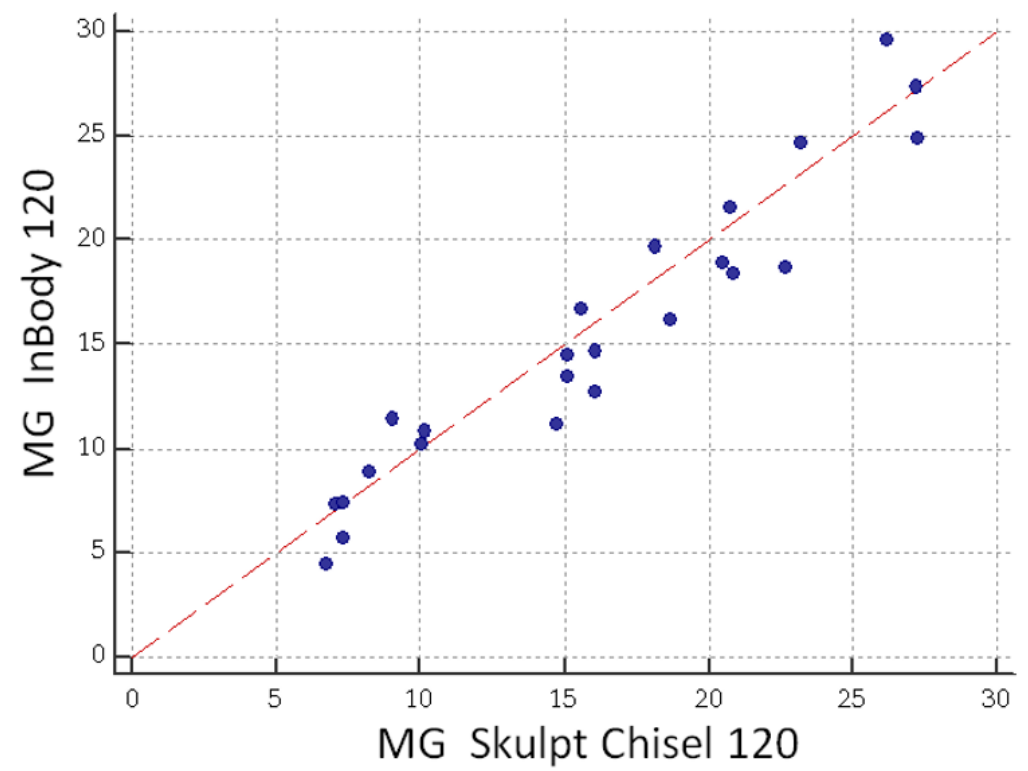

Gráfica 2. Concordancia entre InBody 120 y Skulpt Chisel para las mediciones de MG. 
Los gráficos de Bland-Altman mostraron una a -6,9\%, Gráfica 3) y para MG (diferencia media= diferencia de las medias relativamente pequeña $-0,6 \mathrm{~kg}, I C 95 \%=3,2$ a $-4,4 \mathrm{~kg}$, Gráfica 4) medida por para, el $\% \mathrm{GC}$ (diferencia media= $-0,9 \%, \mathrm{IC} 95 \%=5,1$ ambos instrumentos.

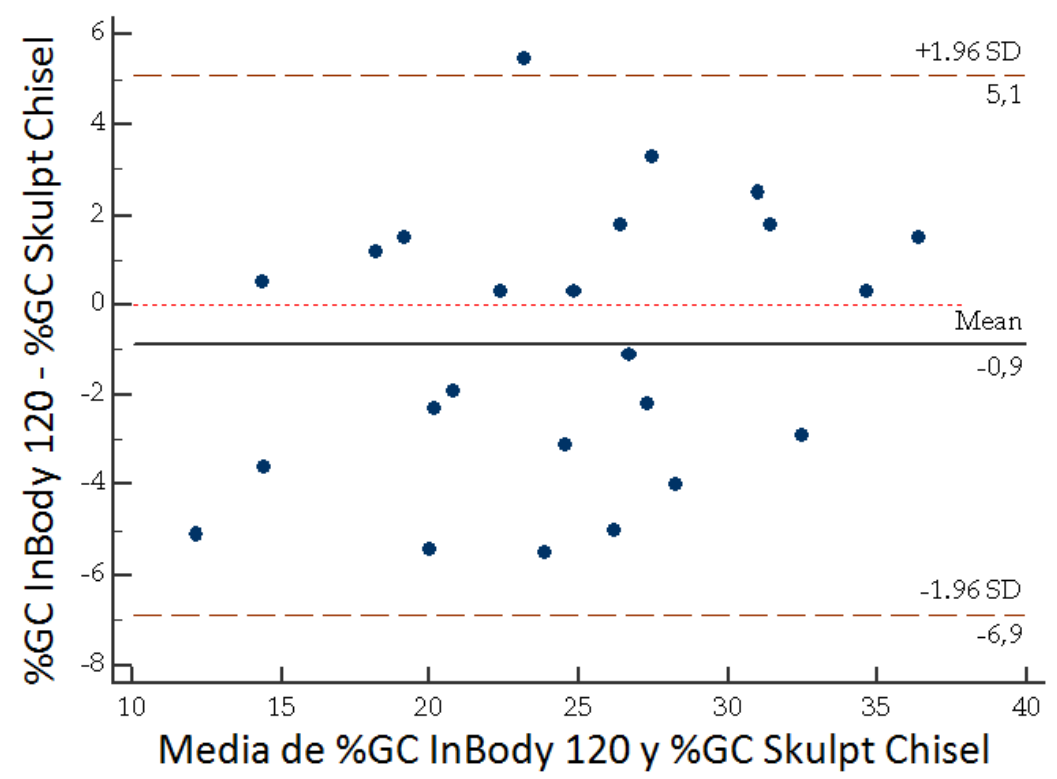

Gráfica 3. Concordancia entre InBody 120 y Skulpt Chisel para el \%GC.

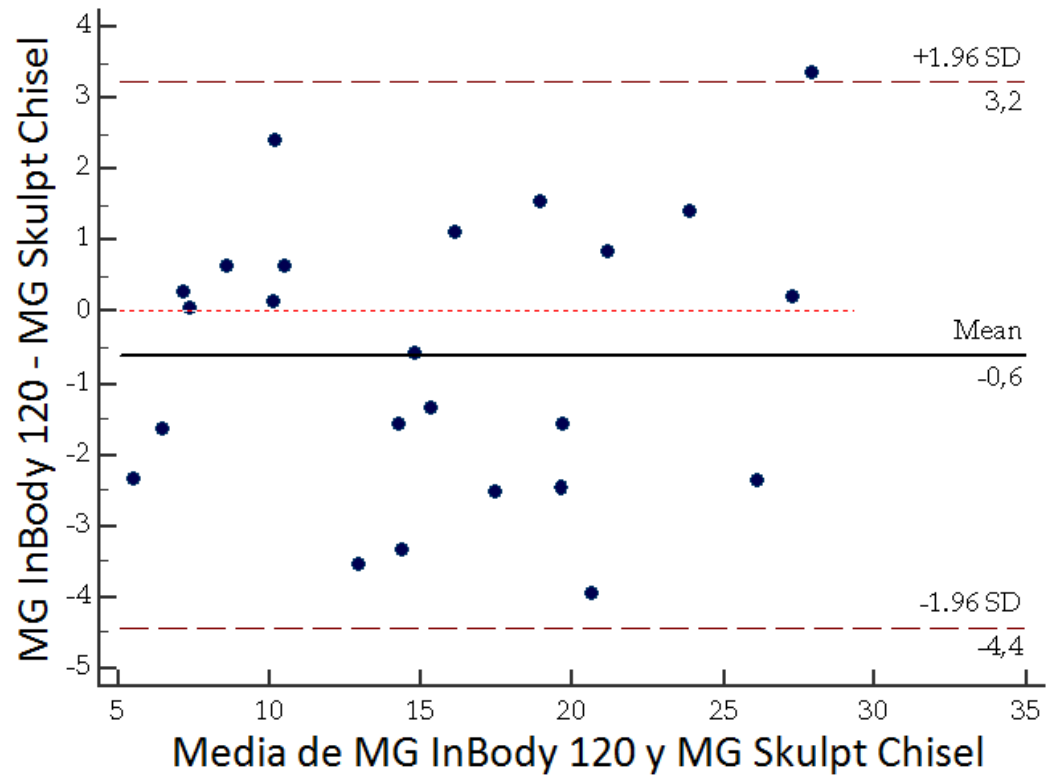

Gráfica 4. Concordancia entre InBody 120 y Skulpt Chisel para MG. 
Además, se encontró una correlación positiva significativa entre los dos instrumentos para la determinación del \%GC $(r=0,898, p<0,01)$ y MG $(r=0,959, \quad p<0,01) . \quad C M \quad$ correlacionó significativamente con, el \%MM $(r=0,771, p<0,01)$, pero no con $\mathrm{MM}(r=-0,116, p=0,589)$. Todas las mediciones del \%GC en la parte superior derecha e izquierda del cuerpo realizadas con Skulpt Chisel se correlacionaron significativamente con \%GC en la extremidad superior derecha $(r=0,562$ a 0,815 , $p<0,01)$ e izquierda $(r=0,560$ a $0,806, p<0,01)$ medido por InBody 120. Lo mismo para las mediciones del porcentaje de grasa abdominal $(r=0,796$ a 0,835, $p<0,01)$. Las mediciones de \%GC en la parte inferior derecha se correlacionaron significativamente con $\%$ GC en la extremidad inferior derecha $(r=0,469$ a $0,612, p<0,05)$, menos los isquiotibiales. Todas las mediciones del \%GC en la parte inferior izquierda se correlacionaron de manera positiva significativa con el \%GC en la extremidad inferior izquierda ( $r=0,587$ a $0,684, p<0,01$ ) (imagen 1).
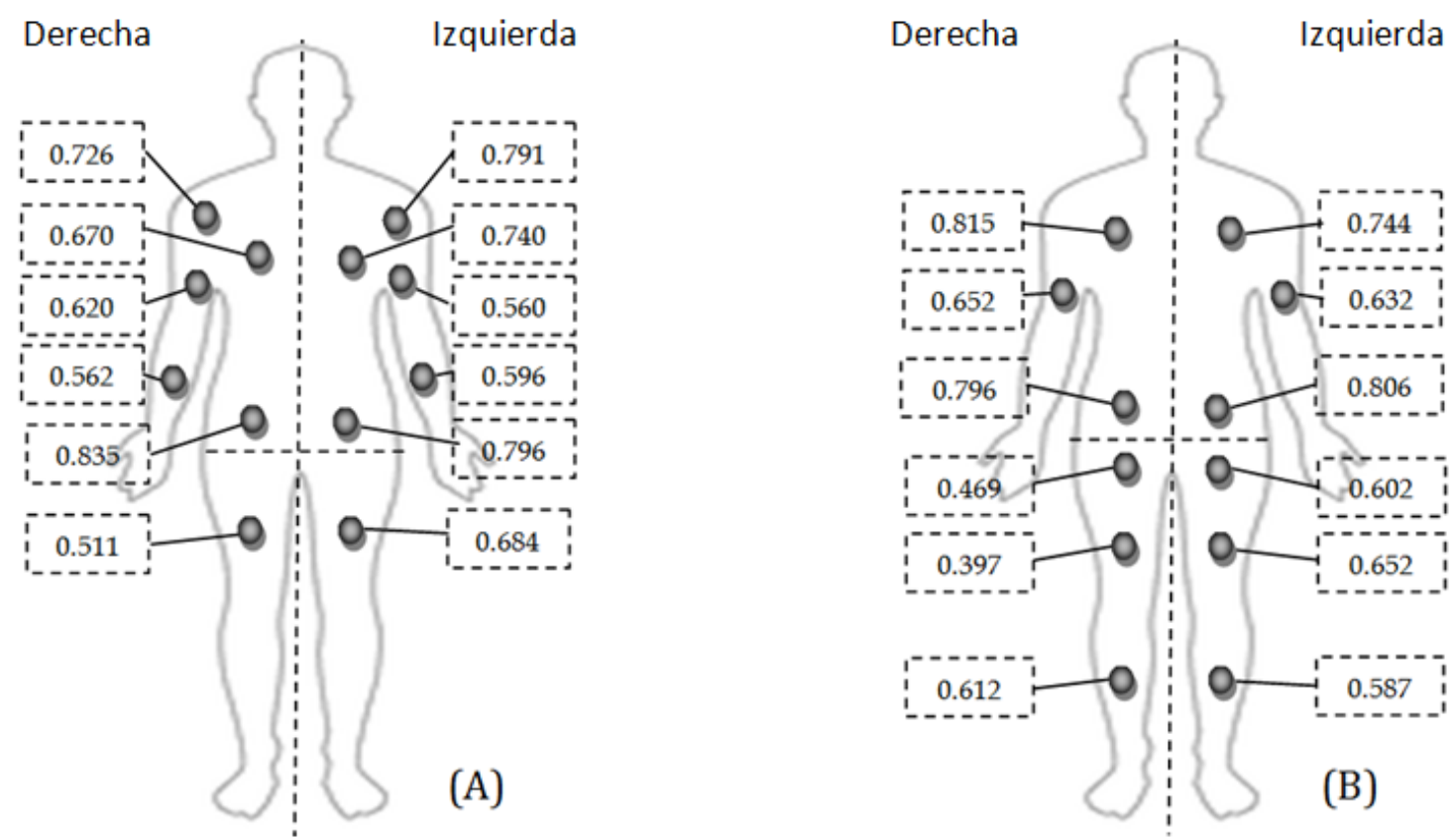

Imagen 1. Correlación entre el porcentaje de grasa por sección

(Skulpt Chisel) y el porcentaje de grasa segmental (InBody 120)

anterior $(\mathrm{A})$ y posterior $(\mathrm{B})$.

Las mediciones de $\mathrm{CM}$ realizadas por Skulpt Chisel en los bíceps, tríceps, antebrazo y hombro derecho se correlacionaron significativamente con el \%MM superior derecho $(r=0,411$ a $0,590, p<0,05)$ medido por el InBody 120, sin observar esto con las mediciones del pecho y la espalda alta y baja. Las mediciones de CM de bíceps, tríceps y antebrazo izquierdo se correlacionaron significativamente con el \%MM superior izquierdo $(r=0,517$ a $0,595, p<0,01)$, pero no con las mediciones de hombro, pecho y espalda alta y baja. El \%MM abdominal no se correlacionó con la
CM abdominal. La CM de los cuádriceps y el glúteo derecho se correlacionaron de manera positiva significativa ( $r=0,483$ y 0,462 respectivamente, $p<0,05)$ con el \%MM inferior derecho, pero no con la pantorrilla y los isquiotibiales. Por otro lado, la CM de los cuádriceps y la pantorrilla izquierda se correlacionaron de manera positva significativa $(r=0,420$ y 0,490 , respectivamente, $p<0,05)$ con el $\% M M$ inferior izquierdo, pero no con los glúteos y los isquiotibiales (imagen 2). 


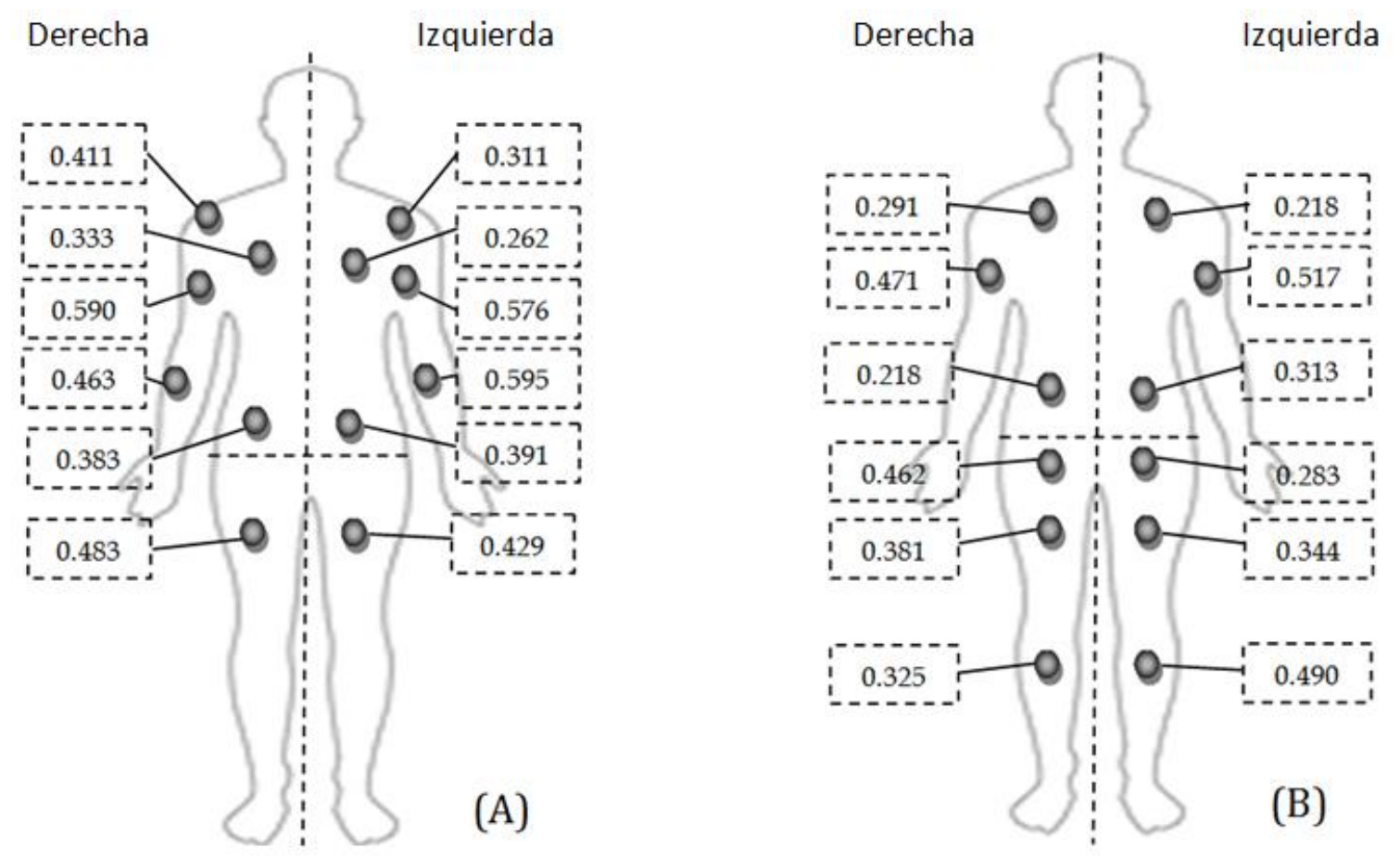

Imagen 2. Correlación entre la calidad muscular por sección

(Skulpt Chisel) y el porcentaje de masa muscular segmental

(InBody 120) anterior (A) y posterior (B).

\section{DISCUSIÓN}

Se encontró en una muestra de atletas adolescentes de combate una concordancia pobre entre los instrumentos para la determinación del porcentaje de grasa corporal. Estos hallazgos pueden explicarse, en parte, a las características de fabricación de ambos instrumentos (Skulpt Performance Training System, 2019; InBody, 2019). El InBody, es un analizador octopolar que emplea dos frecuencias $(20 \mathrm{kHz}$ y $100 \mathrm{kHz}$ ) para la determinación de la composición corporal, mientras que el Skulpt Chisel no especifica el tipo y cantidad de frecuencias utilizadas para las mediciones. Otro aspecto importante, es que a diferencia del InBody 120, el Skulpt Chisel no necesita: 1.- El peso, 2.- Talla y 3.- Género de los sujetos para el cálculo de sus algoritmos, debido a que, de acuerdo a la descripción del fabricante, el instrumento realiza sus cálculos de acuerdo a las características eléctricas de cada tejido. InBody utiliza electrodos de contacto directo para los pies y las manos, mientras que, el Skulpt Chisel se pone en contacto directo con la piel de la zona que será medida; además dicha zona debe de ser hidratada previamente, con la aplicación de agua mediante un atomizador.
Por otra parte, la práctica deportiva de las disciplinas de combate se caracteriza por el contacto físico y el alto impacto (Menéndez, 2017). Las contusiones musculares que se pueden presentar como consecuencia de un traumatismo muscular externo, se caracterizan por la infiltración de sangre y la presencia de edemas (Aguilar, 2005), las mismas que podrían afectar la conductividad del tejido de una zona específica que es medida por contacto directo, como sucede cuando se utiliza el Skulpt. Sin embargo, los atletas respondieron un cuestionario, especificando la ausencia de lesiones, además fueron evaluados en ropa interior, por lo que pudimos asegurarnos que las zonas donde se aplicó el Skulpt Chisel no tuvieran hematomas.

Otro factor que pudo haber influido en los resultados fue el hecho, que la muestra incluyó sujetos con sobrepeso $(n=5, \% 21)$ y obesidad $(n=7,29 \%)$, de acuerdo al IMC para la edad (De Onis et al. 2007). Sin embargo, el IMC no es un indicador recomendado para evaluar a los atletas debido a que pueden presentar una masa muscular acrecentada (Biljana et al. 2012). Se encontró que cinco de los sujetos con IMC normal tenían un \%GC elevado y dos de los 
que tenían un IMC elevado tenían un \%GC normal, de acuerdo a las mediciones realizadas por el InBody 120. De manera similar, cuatro de los sujetos con un IMC normal presentaron un \%GC elevado, de acuerdo a las mediciones realizadas por el Skulpt Chisel. Esto parece evidenciar que el IMC tampoco, es un buen indicador que permita identificar a los adolescentes con un IMC normal y una adiposidad elevada.

También, se pudo observar que no hubo diferencias significativas en la edad, peso, talla, IMC y \%GC total y segmentario entre los atletas de ambas disciplinas. Sin embargo, si se encontró que los luchadores, presentaron un mayor \%MM en el tren superior, de acuerdo a las mediciones del InBody 120. Además, de acuerdo al Skulpt Chisel, los atletas de taekwondo presentaron un mayor \%GC total y por zona (13/24 mediciones) y una menor CM total y por zona (15/24 mediciones). Estas diferencias parecen ser generadas por la práctica continua de las diferentes modalidades de los deportes de combate, porque, el taekwondo se caracteriza por la ejecución de patadas con alto grado de espectacularidad, generando un desarrollo muscular del tren inferior; mientras que, los atletas de luchas realizan proyecciones y llaves, lo que puede propiciar un desarrollo muscular más generalizado (Betancourt et al. 2011).

De acuerdo a los datos de ambos instrumentos todos los sujetos presentaban un \%GC, por encima de los valores mínimos recomendados para atletas adolescentes. Sin embargo $62.5 \%(n=15)$ y $66.6 \%(n=16)$ tuvieron valores por encima de las recomendaciones máximas para adolescentes sanos; de acuerdo a las mediciones realizadas por: el InBody 120 y el Skulpt Chisel de manera respectiva (McArdle, 2009). Además, ambos instrumentos identificaron por igual a los sujetos con exceso de grasa corporal. El exceso de adiposidad que presentaron nuestros sujetos pudo ser un factor que influyera en la pobre concordancia encontrada entre los instrumentos, debido a que, el único estudio disponible en la literatura que ha evaluado la concordancia del Skulpt Chisel con un método de referencia (DXA) trabajó con una muestra que incluía varones jóvenes adultos (?]=24,3 $\pm 4,6$ años) con un \%GC menor al reportado por nosotros (? $=17,5 \pm 6,0 \%$ ) (McLester et al. 2018). Sin embargo, es importante mencionar, que a pesar que los autores concluyeron que había similitud entre las mediciones hechas por ambos instrumentos, basándose en la comparación de medidas, como los límites de concordancia observados en los gráficos de Bland-Altman, que eran clínicamente importantes (+/-6\% aproximadamente) y parecidos a los que se encontraron en este trabajo, evidenciando que las mediciones del \%GC de ambos instrumentos son discordantes (Cortés-Reyes et al. 2009).

En este estudio, a pesar de que se encontraron correlaciones elevadas entre el \%GC y la MG totales y segmentarios; el coeficiente de correlación concordancia de Lin mostró una concordancia pobre entre los dos instrumentos. Además, los gráficos de Bland-Altman mostraron una diferencia de media pequeña, pero la variación individual fue clínicamente relevante en algunos sujetos (hasta $\pm 5 \%$ $y \pm 3 \mathrm{~kg}$ ), evidenciando que no son intercambiables sus mediciones.

De acuerdoa losfabricantes, elSkulptChisel determina la CM mediante miografía por: bioimpedancia eléctrica (EIM) (Skulpt Performance Training System, 2019). Este método fue desarrollado en el año 2000 por Aaron \& Shiffman (2016) con el fin de evaluar grupos musculares de una forma no invasiva; se caracteriza por evaluar un pequeño volumen corporal aplicando corriente externa constante al organismo y evaluando los patrones de resistencia registrados en la superficie, generando patrones eléctricos que describen el estado del músculo. Alteraciones como la atrofia, pérdida de fibras musculares, edema o la infiltración de grasa pueden provocar cambios en las propiedades eléctricas del músculo (Rutkove \& Darras, 2013). Este método ha demostrado ser de utilidad para el diagnóstico, seguimiento y evaluación de la respuesta al tratamiento en personas con enfermedades neuromusculares (Rutkove, 2009; Rutkove et al. 2012; Shiffman, 2013; Ching et al. 2013). Además, debido a que es una técnica no invasiva, de bajo costo, fácil de usar, indolora y que requiere de la participación pasiva del paciente; resulta ser bastante atractiva para la práctica clínica (Colina et al. 2016).

Debido a que, el Skulpt Chisel no permite cuantificar la masa muscular, quisimos conocer, sí, existía relación entre la $\mathrm{CM}$ medida por este instrumento y el \%MM medido por el InBody 120 . Encontramos una correlación positiva y significativa entre estas dos variables. Sin embargo, esto no se presentó, cuando la MM se expresó en kilogramos. De manera similar, la CM de algunas zonas superiores, centrales 
e inferiores del cuerpo no se correlacionaron con él \%MM. Esto parece indicarnos, que la CM es un indicador que puede ayudar a monitorear los cambios cuantitativos y cualitativos, de la masa muscular total, pero, que podría diferir con el análisis segmentario de los métodos de BIA.

\section{CONCLUSIONES}

Entre las debilidades de nuestro estudio podemos mencionar el tamaño de la muestra, la no inclusión de mujeres, la falta de realización de pruebas de laboratorio y gabinete para confirmar que los sujetos fueran sanos. Además, tampoco, se utilizó un método indirecto como referencia para comparar cada uno de nuestros instrumentos y la inclusión de sujetos con adiposidad acrecentada.

El presente estudio es el único en la literatura que compara las mediciones del Skulpt Chisel, con un equipo de impedancia bioeléctrica de la marca InBody. Si bien, ambos son métodos doblemente indirectos, los instrumentos de la marca InBody han mostrado buena concordancia con métodos indirectos de referencia en poblaciones de atletas y sujetos físicamente activos (Utter \& Lambeth, 2010; Miller et al. 2016; Montgomery et al. 2017), por lo que, se puede considerar como un buen método de referencia. Además, tampoco, hay estudios que hayan valorado la concordancia entre estos dos instrumentos en una población de adolescentes físicamente activos. Por lo tanto, consideramos que es necesario realizar más trabajos que permitan un mejor conocimiento sobre los nuevos analizadores de composición corporal, para poder orientar a los clínicos y realizar inversiones inteligentes.

Conflicto de intereses: El manuscrito fue elaborado y revisado por todos los autores, que declaran la ausencia de cualquier conflicto que puede poner la validez de los resultados en riesgo.

Financiamiento: Este trabajo no recibió ningún financiamiento.

\section{REFERENCIAS}

1. AARON, R.; SHIFFMAN, C. 2006. Using Localized Impedance Measurements to Study Muscle Changes in Injury and Disease. Ann N Y Acad Sci. 904(1):171-180.

https://doi.org/10.1111/j.1749-6632.2000. tb06443.x
2. AGUILAR, L. 2005. Traumatismos y tendinitis de las articulaciones. Offarm. 24(3):60-68.

3. ALVERO, J.; CORREAS, L; RONCONI, M.; FERNÁNDEZ, R.; MANZAÑIDO, P. 2011. La bioimpedancia eléctrica como método de estimación de la composición corporal: normas prácticas de utilización. Rev Andal Med Deporte. 4(4):167-174.

4. BETANCOURT, H.; SALINA, O.; ARÉCHIGA, J. 2011. Análisis cineantropométrico de la volumetría muscular de atletas de alto rendimiento de deportes olímpicos de combate. An Antrop. 45:113-122.

5. BILJANA, S.; BORISLAV, O.; GORAN, D.; STOKIC, E.; SINISA, B. 2012. Relationship between body mass index and body fat in children-Age and gender differences. Obes Res Clin Pract. 6(2):167-173. https://doi.org/10.1016/j.orcp.2011.08.153

6. CHING, C.; CHEN, Y.; LU, L.; HSIEH, P.; HSIAO, C.; SUN, T.; SHIEH, H.; CHANG, K. 2013. Characterization of the muscle electrical properties in low back pain patients by electrical impedance myography. PLoSOne. 8(4):e61639. https://doi.org/10.1371/journal.pone.0061639

7. COLINA, E.; GONZÁLEZ, C.; MIRANDA, D. 2016. Miografía por impedancia eléctrica. Rev Col Med Fis Rehab. 26(1):38-49. http://dx.doi.org/10.28957/rcmfr.v26n1a4

8. CORTÉS-REYES, E.; RUBIO-ROMERO, J.; GAITÁNDUARTE, H. 2009. Métodos estadísticos de evaluación de la concordancia y la reproducibilidad de pruebas diagnósticas. Rev Colomb Obstet Ginecol. 61(3):247-255.

9. COSTA, O.; ALONSO, D.; PATROCINIO, C.; CANDIA, R.; DE PAZ, J. 2015. Métodos de evaluación de la composición corporal: una revisión actualizada de descripción, aplicación, ventajas y desventajas. Arch Med Deporte. 32(6):387-394.

10. DE ONIS, M.; ONYANGO, A.; BORGHI, E.; SIYAM, A.; NISHIDA, C.; SIEKMANN, J. 2007. Development of a WHO growth reference for school-aged 
children and adolescents. Bull World Health Organ. 85(9):660-667.

https://doi.org/10.2471/blt.07.043497

11. FRANCHINI, E.; NUNES, A.; MORAES, J.; DEL VECCHIO, F. 2007. Physical fitness and anthropometrical profile of the Brazilian male judo team. J Physiol Anthropol. 26(2):59-67. https://doi.org/10.2114/jpa2.26.59

12. GARROW, J. 1982. New approaches to body composition. Am J Clin Nutr. 35(5 Suppl):1152-1158. https://doi.org/10.1093/ajcn/35.5.1152

13. INBODY. 2019. InBody 120. Inbodylatinamerica. com. Disponible desde Internet en: http://www.inbodylatinamerica.com/ Modelos/inbody_120

14. LEE, S.; GALLAGHER, D. 2008. Assessment methods in human body composition. Curr Opin Clin Nutr Metab Care. 11(5):566-572.

15. LIN, L. 1989. A concordance correlation coefficient to evaluate reproducibility. Biometrics. 45(1):255268.

16. LUSTGARTEN, M.; FIELDING, R. 2011. Assessment of analytical methods used to measure changes in body composition in the elderly and recommendations for their use in phase II clinical trials. J Nutr Health Aging. 15(5):368375.https://doi.org/10.1007/s12603-011-0049-x

17.MARFELL-JONES, M.;STEWART,A.;CARTER,J. 2006. International standards for anthropometric assessment. in UNSW Press:Sydney.

18. MATA-ORDÓÑEZ, F.; SÁNCHEZ-OLIVER, A.; DOMÍNGUEZ-HERRERA, R. 2018. Importancia de la nutrición en las estrategias de pérdida de peso en deportes de combate. J Sport Health Res. 10(1):1-12.

19. MATTSSON, S.; THOMAS, B. 2006. Development of methods for body composition studies. Phys Med Biol. 51(13):R203-228. https://doi.org/10.1088/0031-9155/51/13/r13
20. MCARDLE, W.; KATCH, F.; KATCH, V. 2009. Sports and Exercise Nutrition. Lippincott Williams \& Wilkins. 720p.

21. MCLESTER, C.; DEWITT, A.; ROOKS, R.; MCLESTER, J. 2018. An investigation of the accuracyand reliability of body composition assessed with a handheld electrical impedancemyography device. Eur J Sport Sci. 18(6):763-771. https://doi.org/10.1080/17461391.2018.144 8458

22. MENÉNDEZ, J. 2017. Las artes marciales y deportes de combate en educación física. Una mirada hacia el kickboxing educativo. Revista Digital de Educación Física. 48:108-119.

23. MESA, L.; GARCÍA, T.; LINARES, F.; AGUILERA, B. 2015. Caracterización de la composición corporal de las atletas de taekwondo del estado Cojedes en el periodo de preparación general. Cuadernos de Psicología del Deporte. 12(211):89-94.

24. MILLER, R.; CHAMBERS, T.; BURNS, S. 2016. Validating InBody ${ }^{\circledR} 570$ Multi-frequency Bioelectrical Impedance Analyzer versus DXA for Body Fat Percentage Analysis. JEP on line. 19(5):71-78.

25. MONTGOMERY, M.; MARTTINEN, R.; GALPIN, A. 2017. Comparison of Body Fat Results from 4 Bioelectrical Impedance Analysis Devices vs. Air Displacement Plethysmography in American Adolescent Wrestlers. IJKSS. 5(4):18-25. http://dx.doi.org/10.7575/aiac.ijkss.v.5n.4p.18

26. NAVALTA, J.; STONE, W.; LYONS, T. 2019. Ethical Issues Relating to Scientific Discovery in Exercise Science. International Journal of Exercise Science. 12(1):1-8.

27. PENICHE, C.; BOULLOSA, B. 2011. Nutrición aplicada al deporte. McGraw-Hill (México). $386 p$.

28. PORBÉN, S.; BORRÁS, A. 2003. Composición corporal. Acta Médica. 11(1):26-37. 
29. RUTKOVE, S. 2009. Electrical Impedance Myography: Background, Current State, and Future Directions. Muscle Nerve. 40(6):936-946. https://dx.doi.org/10.1002\%2Fmus.21362

30. RUTKOVE, S.; CARESS, J.; CARTWRIGHT, M.; BURNS, T.; WARDER, J.; DAVID, W.; GOYAL, N.; MARAGAKIS, N.; CLAWSON, L.; BENATAR, M.; USHER, S.; SHARMA, K.; GAUTAM, S.; NARAYANASWAMI, P.; RAYNOR, E.; WATSON, M.; SHEFNER, J. 2012. Electrical impedance myography as a biomarker to assess ALS progression. Amyotroph Lateral Scler. 13(5):439-445.

https://doi.org/10.3109/17482968.2012.688837

31. RUTKOVE, S.; DARRAS, B. 2013. Electrical impedance myography for the assessment of children with muscular dystrophy: a preliminary study. J Phys Conf Ser. 434(1):1-8. https://doi.org/10.1088/1742-6596/434/1/012 069

32. SANT'ANNA, M.; PRIORE, S.; FRANCESCHINI, S. 2009. Métodos de avaliação da composição corporal emcrianças. Rev Paul Pediatr. 27(3):315-321. http://dx.doi.org/10.1590/ S0103-05822009000300013

33. SHIFFMAN, C. 2013. Circuit modeling of the electrical impedance part III: Disuse following bone fracture. Physiol Meas. 34(5):487-502. https://doi.org/10.1088/0967$3334 / 34 / 5 / 487$

34. SKULPT PERFORMANCE TRAINING SYSTEM. 2019. How sculpt compares. Disponible desde Internet en: https://www.skulpt.me/body_fat

35. TABBEN, M.; CHAOUACHI, A.; MAHFOUDHI, M.; ALOUI, A.; HABACHA, H.; TOURNY, C.; FRANCHINI, E. 2014. Physical and physiological characteristics of high-level combat sport athletes. J Sport Health Res. 5(1):1-5.

36. THIBAULT, R.; GENTON, L.; PICHARD, C. 2012. Body composition: why, when and for who? Clin Nutr. 31(4):435-447.
37. UTTER, A.; LAMBETH, P. 2010. Evaluation of multifrequency bioelectrical impedance analysis in assessing body composition of wrestlers. Med Sci Sports Exerc. 42(2):361-367. https://doi.org/10.1249/mss.0b013e3181b2e8b4

38. WANG, Z.; PIERSON, R.; HEYMSFIELD, S. 1992. The five-level model: a new approach to organizing body-composition research. Am J Clin Nutr. 56(1):19-28. https://doi.org/10.1093/ajcn/56.1.19 